\title{
Pre-transplant ventricular assist device explant
}

\author{
Espeed Khoshbin, Stephan Schueler
}

The Institute of Transplantation, Freeman Hospital, Newcastle Upon Tyne, UK

Correspondence to: Espeed Khoshbin. The Institute of Transplantation, Freeman Hospital, Newcastle Upon Tyne NE77DN, UK.

Email: Espeed.Khoshbin@nuth.nhs.uk.

\begin{abstract}
Explantation of a left ventricular assist device (LVAD) may be challenging even in the most experienced hands. We aim to describe the technique for explantation of an LVAD together with the heart as applicable to all contemporary implantable mechanical assist devices. In order to ensure safe explantation, particular care must be taken at three distinct stages: at the time of LVAD implantation, at pre-transplant assessment and at the time of heart transplantation. The preparation for a safe explantation at LVAD implantation includes positioning the driveline and the outflow graft away from the back of the sternum to ensure protection from injury during re-entry into the chest. At transplant assessment, essential investigations include computed tomography (CT) of the chest and ultrasound imaging of femoral vessels. At the time of heart transplantation, the site of peripheral access should be prepared and vessels exposed in case of a need for emergency bypass. We advise careful dissection starting from the lower aspect of the under surface of the sternum, moving as proximally as possible before attempting to use the oscillating saw. Much of the dissection of the heart is done off-pump. Cardiopulmonary bypass may be established either through peripheral vessels or the outflow graft in an emergency. Central direct cannulation is then established. After the heart and major vessels are isolated, explantation of the heart may begin either en-bloc or after splitting the ventricles in a sagittal plane. The basal regions of both ventricles and both atria are removed, leaving generous cuffs for anastomosis of the left atrium, pulmonary artery, aorta, inferior and superior vena cava (SVC). The apex of the heart is then removed with the device taking care not to injure the phrenic nerve.
\end{abstract}

Keywords: Mechanical assist device; heart transplantation; device explantation

Submitted Aug 16, 2017. Accepted for publication Dec 28, 2017.

doi: $10.21037 /$ acs.2018.01.04

View this article at: http://dx.doi.org/10.21037/acs.2018.01.04

\section{Introduction}

HeartWare left ventricular assist device (HVAD) and St. Jude HeartMate III are the most contemporary implantable left ventricular assist devices (LVADs) used to bridge patients to cardiac transplantation. A centrifugal pump head directs the flow of blood from the left ventricular apex, into an outflow tract which traverses across the base of the heart and is then directed upwards to drain into its confluent with the ascending aorta. The pump is powered by current supplied through a driveline. The driveline is tunnelled in close proximity to the deep fascia over the abdominal wall and brought out through the skin above the patient's waist line. In this article we consider explantation of this type of ventricular assist device.
Continuous-flow LVAD has become the standard of care for patients with end-stage heart failure as a bridge to transplantation (1). However, compared to first-time sternotomy, LVAD explantation may be challenging for a number of reasons. These include the presence of fibrous adhesions encasing the device, position of the outflow tract and the driveline in relation to the sternum (Figure 1) and the possibility that peripheral access may be too narrow for bypass secondary to vascular girth regression as a result of non-pulsatile blood flow (Figure 2). The art of operative technique for VAD explantation therefore involves prior planning as early as the time of VAD implantation, at the transplant re-assessment and finally intraoperatively at the time of heart transplantation. Each of the above will be discussed below. In this article we describe explantation of 

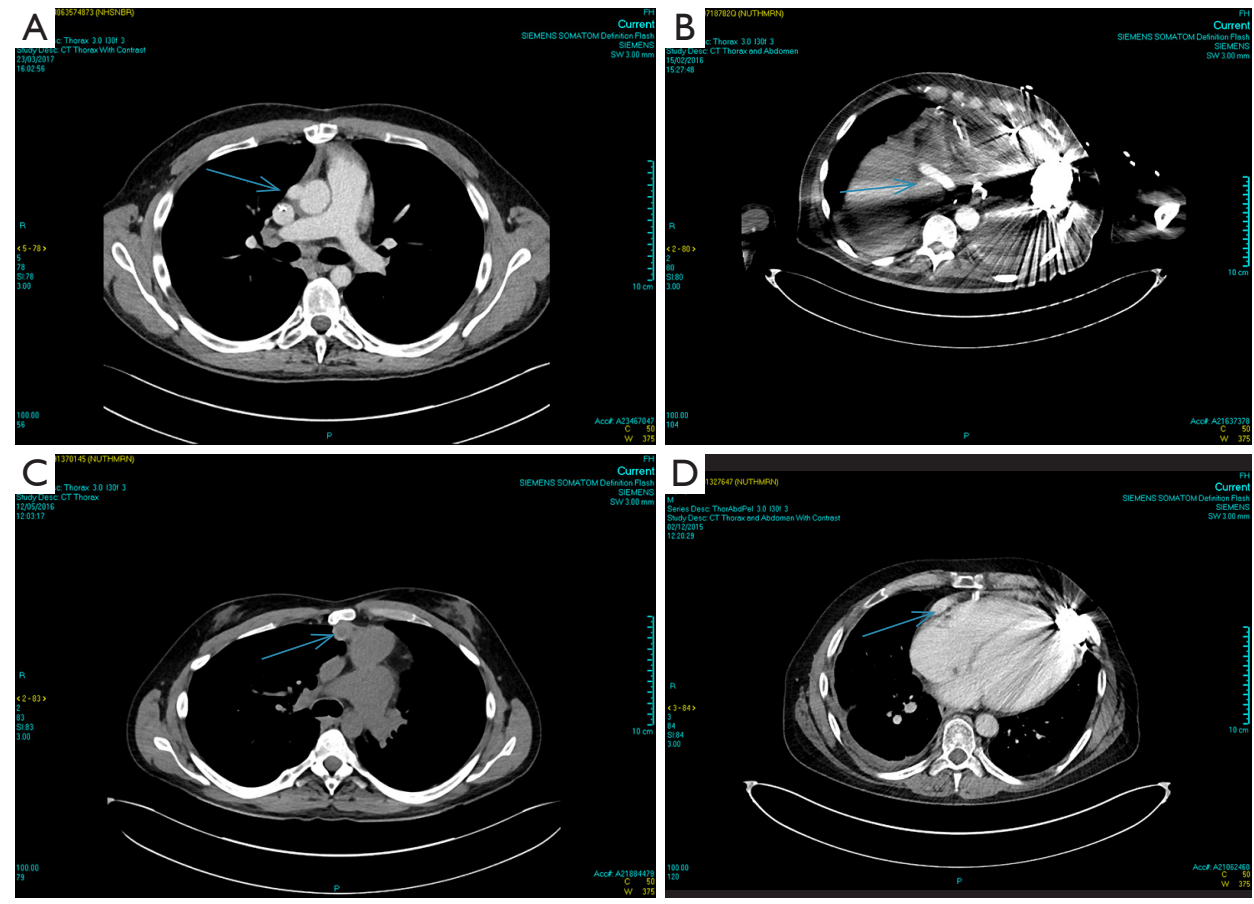

Figure 1 Computed tomography of the thorax. (A) Illustrates desired position of the outflow graft as it joins the ascending aorta laterally; (B) illustrates desired position of the outflow graft at the lower end of the sternum; (C) proximity of the outflow graft to the posterior plate of the upper sternum puts the graft at risk during re-sternotomy; (D) close proximity between the outflow graft and lower sternum putting the graft at risk; the arrows point to the position of the outflow tract or driveline.

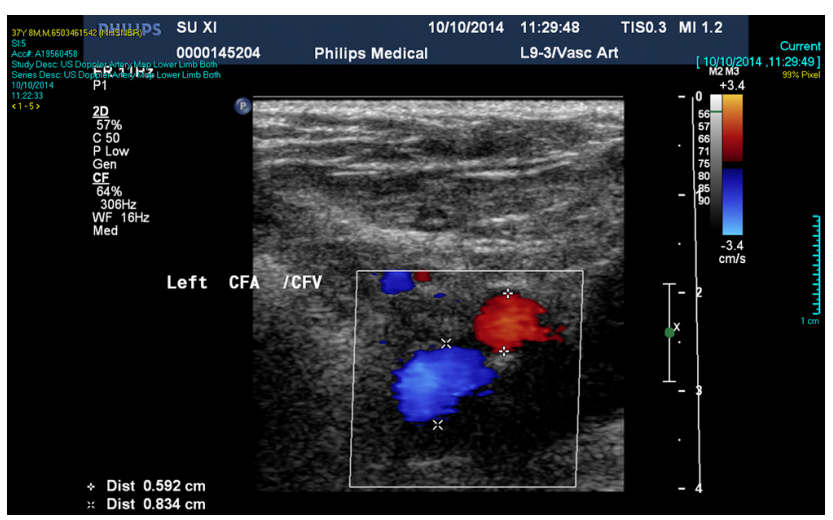

Figure 2 Ultrasound assessments of the common femoral artery. This allows planning for peripheral access. Commonly the artery is of small calibre and may be too small for cannulation on its own to achieve adequate arterial return during cardiopulmonary bypass.

HVAD. The same principles may be applied to explantation of other commonly used implantable devices such as the HeartMate II and III.

\section{Operative techniques}

\section{At implantation}

Preparation for a safe VAD explantation starts at VAD implantation. There are a number of technical considerations which minimize the risk of injury to the outflow graft and driveline at re-sternotomy. Firstly, the driveline would be at risk during re-sternotomy at the lower end of sternum. Secondly, there are two points along the sternum where the outflow graft most commonly comes in close proximity to the back of the sternum hence making it susceptible to injury during re-sternotomy. These points are at the lower end, where the outflow graft crosses the midline to reach the right side, and at the top, where the outflow graft is sewn to the ascending aorta. Figures 3,4 illustrate how prior planning during implantation can minimize the risk of damage to the driveline and the outflow graft during re-sternotomy. Relevant measures are outlined as follows:

(I) Wrapping the driveline almost once around the pump device diverts its path away from the back 


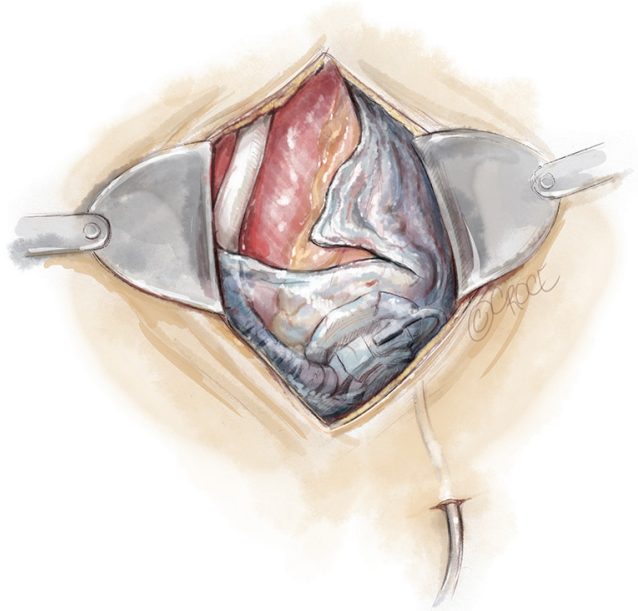

Figure 3 This diagram illustrates how wrapping the driveline around the pump head diverts its path to a safer position away from the midline and the sternum.

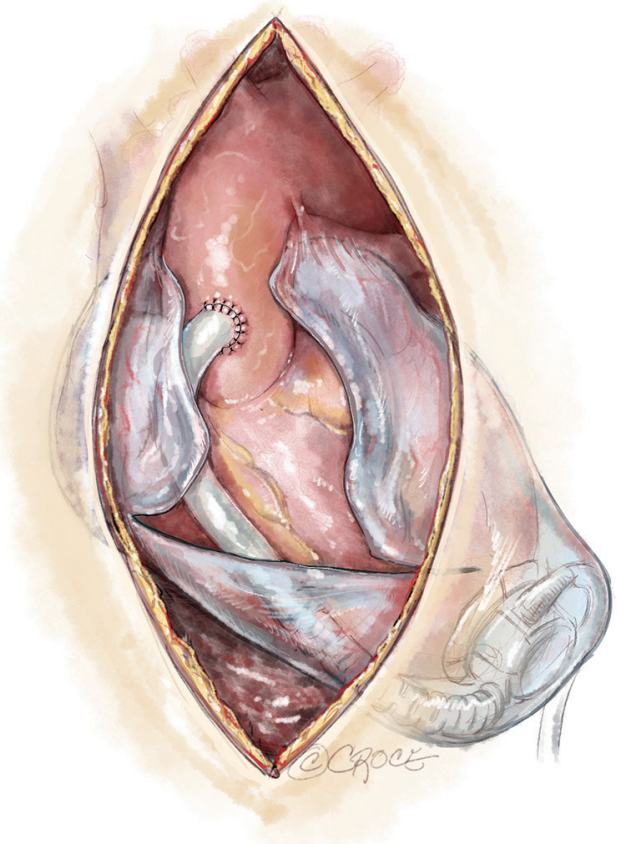

Figure 4 Leaving a ridge of pericardium close to the diaphragm when fashioning the inverted $\mathrm{T}$ incision on the pericardium may reduce the risk of injury to the outflow graft at re-sternotomy.

of the sternum. It will encourage it to exit the pericardium along the left costophrenic angle (Figure 3). The driveline is then tunnelled away to a desired position in the abdominal wall over the

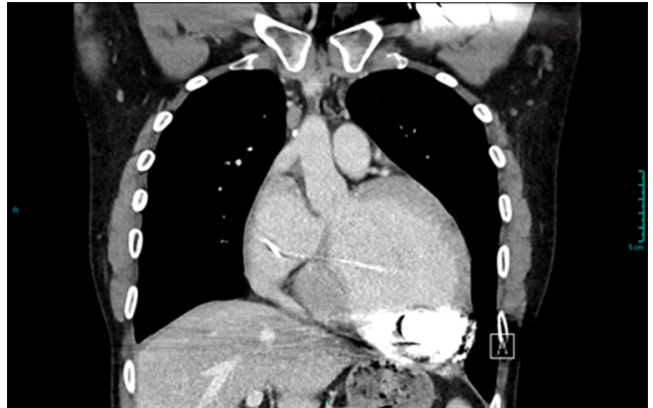

Figure 5 A contrast CT of the thorax showing the position of the graft in relation to the ascending aorta. The outflow graft is sewn to the aorta as laterally as possible on an angle of about 45 degrees to its greater curve on the right-hand side. This is an ideal position away from the path of the oscillating saw in contrast to diagram $1 \mathrm{~b}$.

deep fascia;

(II) A 2-cm ridge of pericardium left intact at the lower end of the sternotomy at the time of VAD implantation will protect the outflow tract. This is formed as part of the inverted $\mathrm{T}$ incision over the pericardium (Figure 4);

(III) The anastomosis of the outflow graft to the ascending aorta should be performed as laterally as possible on the right side to avoid its proximity to the back of the sternum (Figure 5). This may not be possible in a congenital patient due to abnormal morphology which may pose a challenge when positioning the graft away from the sternum;

(IV) Finally, measuring the correct length of outflow graft so that it isn't overly long will prevent its malposition after closure of the sternum.

Polytetrafluoroethylene (PTFE) surgical membrane for pericardial closure has been shown to prevent cardiac injury during re-sternotomy $(2,3)$. Use of PTFE membranes in patients undergoing a bridge to transplantation with an LVAD limits adhesions between tissues and device surfaces without increasing the risk of infection (4-7). Expanded PTFE may be wrapped around surfaces of circulatory support devices in patients being bridged to heart transplantation and the plane of dissection between tissues and surfaces of the mechanical support device is easily discerned (4). There may be no adhesions observed between tissues and membranes facilitating VAD explanation (7). There are a number of PTFE products available in the form of a thin Gore-Tex Pericardial Membrane (Gore Preclude, Gore) or in a tubular form (Gore-Tex Stretch Vascular 


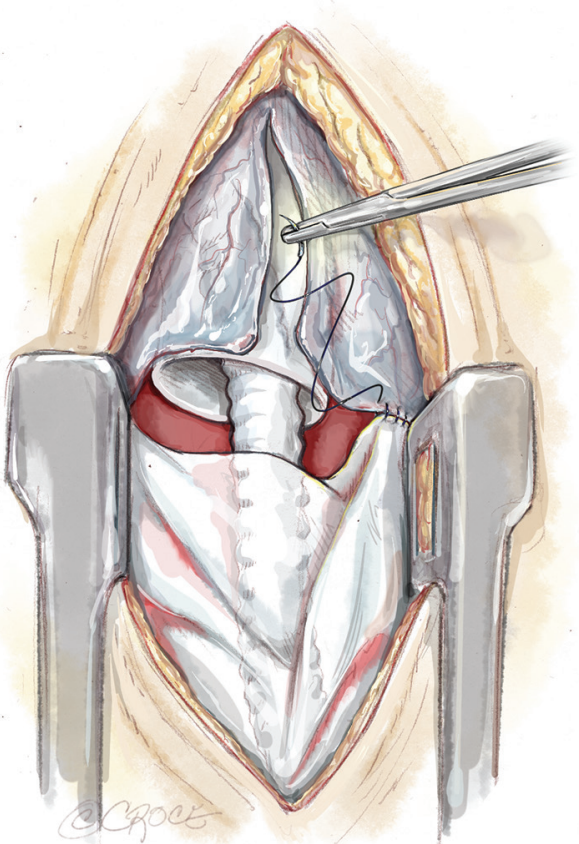

Figure 6 Intraoperative illustration showing both a PTFE tube graft and a Gore-Tex membrane used during implantation in order to minimize adhesion between the heart, outflow graft and sternum. PTFE, polytetrafluoroethylene.

Graft, Gore) protecting the outflow graft from forming dense adhesions to the surrounding tissues. Figure 6 shows utilization of both these products. This practice is more common in our congenital group as discussed above.

\section{At transplant re-assessment}

Prior to re-listing for transplantation, a number of key investigations are necessary. These include computed tomography (CT) imaging of the thorax and ultrasound imaging of the femoral vessels.

A contrast CT scan of the thorax is mandatory for safe re-sternotomy as it demonstrates the proximity of the posterior plate of the sternum to the surface of the heart as well as the position of the driveline and outflow graft in relation to the sternum. Figure 1 illustrates ideal positioning of the driveline and outflow graft in relation to the posterior plate of the sternum in comparison with less ideal positioning.

Ultrasound imaging of the femoral vessels is important as it determines the calibre of the vessels for peripheral vascular access. This is illustrated in Figure 2. Occasionally, safe re-sternotomy necessitates exposure of the groin for cannulation or pre-emptive placement of the patient on peripheral cardiopulmonary bypass prior to re-sternotomy. The latter empties the heart away from the path of the oscillating saw. It may also provide hemodynamic stability in the event of an injury to the driveline or the outflow graft. Other emergency cannulation techniques such as direct cannulation of the outflow graft will be discussed later.

A phenomenon known as vascular girth regression leads to an unusually small calibre of peripheral arterial vessels, such as femoral arteries, rendering them difficult to cannulate especially in an emergency. The pathophysiology of vascular girth regression is thought to relate to nonpulsatile blood flow during prolonged periods of mechanical circulatory support. Prior measurement of the size of both femoral vessels using ultrasound will thus be important in preoperative planning. In cases of bilaterally small femoral vessels, multiple smaller cannulae may be used in both groins and/or the axillary artery in order to obtain adequate return flow. This requires careful surgical planning at the point of assessment and not immediately prior to surgery.

\section{At the time of transplant}

The key to a successful heart transplantation in a patient bridged using an implantable mechanical circulatory device is prior planning. This plan should be relayed to all members of the surgical and anaesthetic team preoperatively.

\section{Preparation}

The general preparation of the patient is the same as that of a standard redo cardiac surgery. It is important not to forget to switch off the implanted defibrillator device prior to surgery and to attach external defibrillator pads to the chest. The patient is prepared with both groins marked using ultrasound imaging ready for cannulation of the common femoral vessels if or when indicated. Occasionally one or both groin vessels have already been used at the time of VAD implantation either for peripheral bypass, for ECMO or a temporary right ventricular assist device (RVAD) after LVAD implantation. Visualisation of the femoral artery is mainly a precautionary measure as, with careful planning of the re-sternotomy, the need for direct femoral cannulation is minimal. The femoral vein may however be used in heart transplantation for venous drainage on bypass to improve access for IVC anastomosis at the time of heart implantation. Use of the long saphenous vein as an entry point to the 


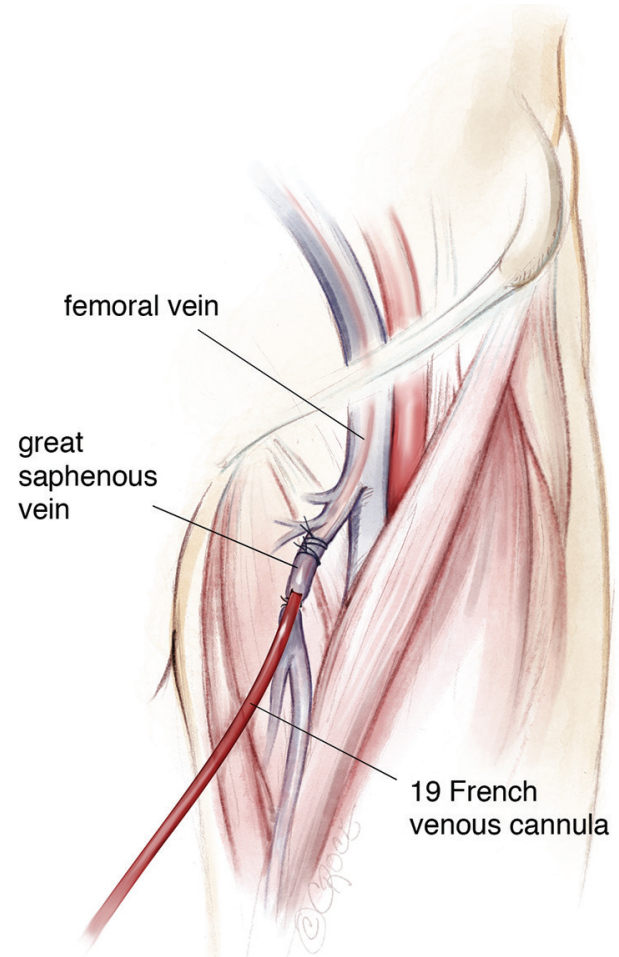

Figure 7 Indirect femoral cannulation through the long saphenous vein. A 19 French venous cannula may be introduced with relative ease in the majority of patients with adequate drainage. The vein is simply tied off on either side of the cannulation site after decannulation at the end of the operation. This minimizes the formation of adhesions in the groin or the risk of narrowing the vessel by using a direct purse string on the vessel.

femoral vein for ECMO or RVAD following LVAD implantation may reduce adhesions in the groin (Figure 7), facilitating subsequent cannulation of the femoral vein at the time of transplant. Using an end-to-side tube graft for femoral arterial cannulation for ECMO on the other hand worsens the presence of adhesions in the redo groin incision. This is especially worse if the remnant of the tube graft is left inside after de-cannulation by simply tying it. We therefore recommend removal of the graft material if possible and reconstruction of the femoral artery at the time of de-cannulation. The infraclavicular regions are also exposed for access when removing the implanted cardiac defibrillator at the end of the operation and for access to the contralateral axillary artery in case of the need for axillary cannulation. The abdomen is also prepared and left exposed for removal of the extra-thoracic portion of the driveline at the end of the operation.

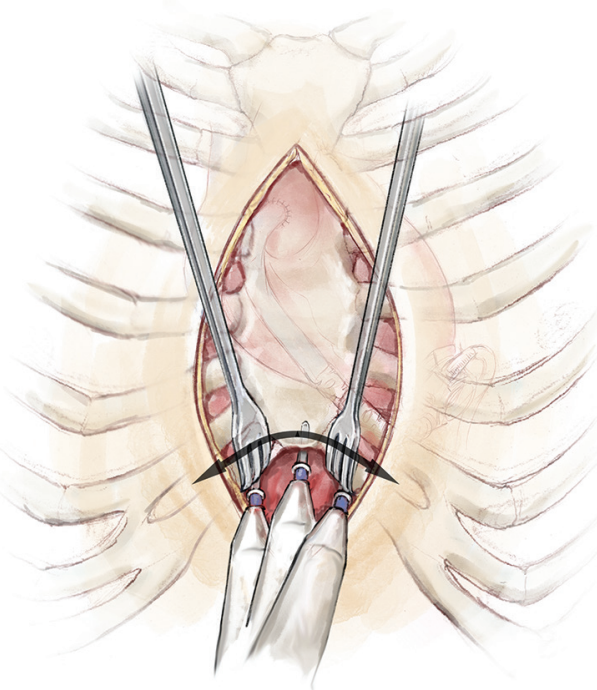

Figure 8 This diagram illustrates a safe method of redo sternotomy by freeing the lower aspect of the under surface of the sternum where the outflow graft frequently is encountered. Inadvertent injury to the outflow graft at this point before the chest is even opened may cause significant harm.

\section{Exposition}

One should always allow ample time for explantation of the heart, hence donor cross-clamp time should be arranged in such a way as to minimize the period of donor organ ischaemia as well as recipient bypass time. When considering the timing of surgery, one should consider the time needed for anaesthesia and preparation as well as surgical explantation of the VAD. It is advisable to allow at least 2 to 3 hours for recipient preparation, including a short but well-deserved break before the arrival of the donor heart. Therefore, the timing of the donor crossclamp should be adjusted accordingly.

\section{Operation}

In the majority of cases, an oscillating saw is used to perform the re-sternotomy. Whether on or off bypass at the time of re-sternotomy, care is taken to avoid injury retrosternal structures. We advise careful dissection starting from the lower aspect of the under surface of the sternum and moving as proximally as possible before attempting to use the saw to maximize safety (Figure 8). The lower end of the sternum is then hooked upwards using an instrument of choice while the anterior plate of the sternum is divided 
first. This is followed by the posterior plate. Leaving the sternal wires intact behind the sternum and only dividing them anteriorly may protect against excess downward penetration and myocardial injury by the saw. After completion of the re-sternotomy and removal of the wires the sternum is freed from the underlying fibrous tissues just enough to allow placement of the sternal retractor. The retractor is slowly cranked open to allow access to the heart. The intra-pericardial dissection is typically commenced at the lower margin of the heart as the planes in this region are better defined. Once in the correct plane the pericardium is lifted off the heart using dissecting scissors. As soon as the outflow graft is identified it can be traced up towards its confluent with the ascending aorta. In this way the right side of the heart is freed of adhesions preferably without cardiopulmonary bypass and heparinization. When the right side of the heart is free, it is then time to free the aorta for bypass cannulation.

Throughout this procedure the groin is kept exposed and ready in case of a need for emergency access. However as soon as the LVAD outflow graft is safely isolated it may be used for direct cannulation. In such an emergency, a 24 French Sorin arterial cannula (Ref: NA4528) may be used to directly cannulate the outflow graft in order to quickly establish cardiopulmonary bypass. During such event, the LVAD is turned off and the proximal part of the outflow tract is tied off before cannulation of the distal graft. Venous drainage may be one of either femoral or direct atrial cannulation until definitive venous cannulation is performed. This provides haemodynamic stability until such time that the aortic cannula is placed high up in the ascending aorta in preparation for heart implantation. At this point the planes on the left side of the heart should be developed on either side of the impellor both inferiorly and laterally as well as anteriorly over the left ventricle. This manoeuver would in the majority of circumstances necessitate cardiopulmonary support with full systemic heparinization. Whether or not bypass is already instigated through the outflow graft, the patient should now be cannulated for definitive cardiopulmonary bypass via a peripheral femoral vein and direct central superior vena cava (SVC) (bi-caval) drainage as well as distal ascending aortic return cannula. If already on peripheral femoral bypass or on bypass through the outflow graft, the bypass cannulae are swapped during a short period of controlled circulatory arrest with the patient cooled to 28-32 degrees. In the authors' opinion, the femoral approach to venous cannulation facilitates
IVC anastomosis during transplantation. Both cavae are now ready for snaring during explanation just prior to application of the aortic cross clamp and after ensuring adequate venous drainage.

\section{Completion}

On bypass, the aorta, pulmonary artery, SVC and inferior vena cava (IVC) are dissected and isolated away from their adjacent structures. A combination of sharp and blunt dissection is then used to deliver the device from the apex of the pericardium. This is similar to explantation of a defibrillator device from its fibrous pocket. The explantation of the heart may be done in one of two ways: either en-bloc or after splitting the ventricles in a sagittal plain. The basal regions of both ventricles and both atria are then removed leaving generous cuffs for anastomosis of the left atrium, pulmonary artery, aorta, inferior and SVC. The former may be done before the application of cross-clamp to the recipient's aorta, i.e., 'the point of no return'. The latter, on the other hand, necessitates aortic cross-clamping but may allow better visibility and better access to the apex of the heart (Figure 9). It is generally recommended not to clamp the aorta until the donor heart is safely in the operating room for the recipient. In either case, when the capsule containing the pump head is reached, diathermy is used to make a sufficiently large incision in the capsule to deliver the device. At this point, the left phrenic nerve is particularly vulnerable and care should be taken not to cut or stretch it. When the top portion of the heart is explanted, the defibrillator leads protruding from the cut edge of the SVC are placed on tension and divided. When the VAD and the apex of the heart are explanted, the driveline is pulled hard in the chest and cut flush with the pericardium. At this stage, meticulous haemostasis is imperative especially at the inferior and apical aspects of the pericardium, which are common sites of postoperative bleeding. Care should be taken not to injure the left phrenic nerve through excessive heat generated by the diathermy. The heart is then implanted in the usual way according to the preference of the surgeon.

The abdominal portion of the driveline is pulled out by sustained traction along the straight line. This is performed at the end of the operation usually after the chest is closed. If the driveline is tunnelled in a zigzag to provide additional tunnelling distance from the exit point to the device, then an incision over the point of angulation is necessary and the driveline should be divided at that point (Figure 10). 
A

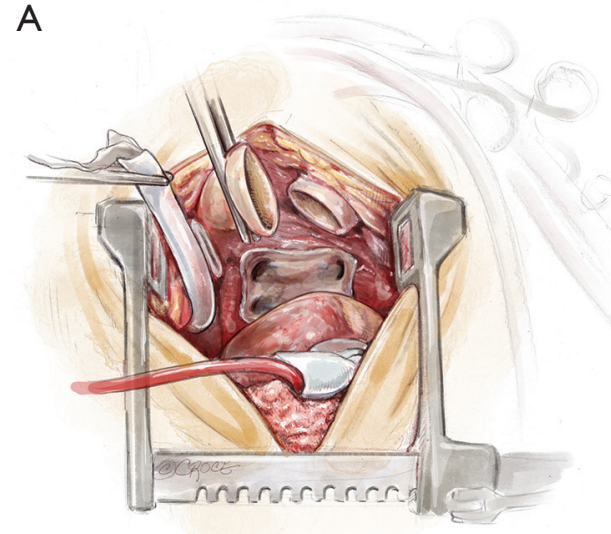

C

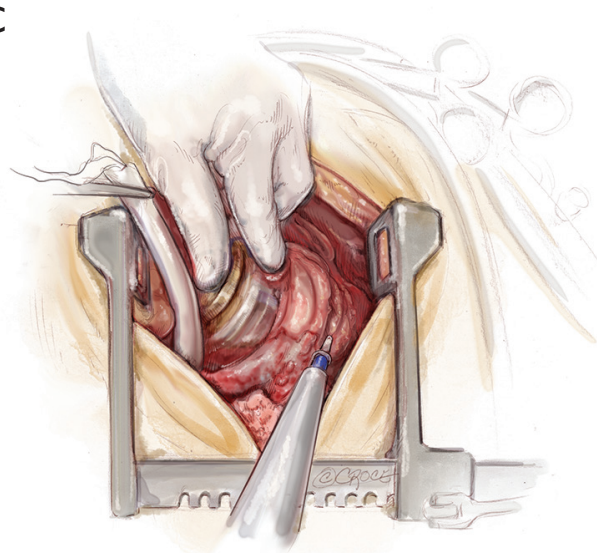

B
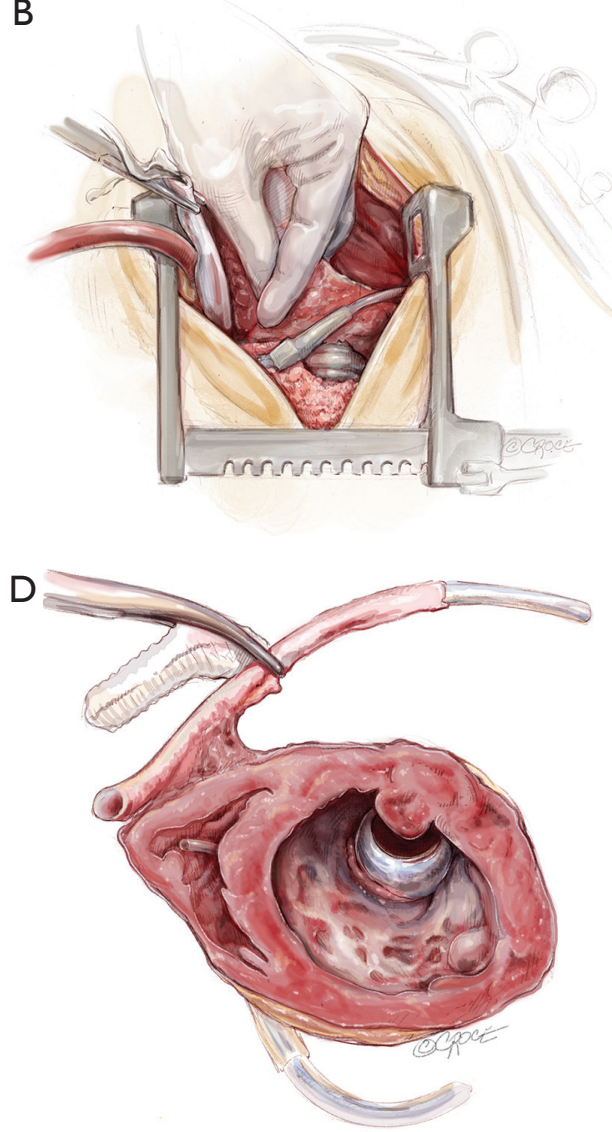

Figure 9 The sequence of device explantation from removal of the top portion of the heart (A) to delivery from the capsule of the device (B), resection at the apex where the pump head resides (C), and finally the cut surface of the ventricles following removal out of the chest (D). The bevel of the pump can be seen from the inside of the left ventricle as it is explanted.

\section{Comments}

\section{Clinical results}

There are no published clinical results on this subject however there are alternative techniques of VAD implantation that may allow comparison if studied in a controlled environment. For example, some alternative techniques avoid re-sternotomy hence allowing a reciprocating saw to be used on a virgin sternum at the time of transplantation. In this technique implantation is performed through bilateral thoracotomies (8). In brief, the patient is placed supine on cardiopulmonary bypass through femoro-femoral cannulation. A left limited anterior thoracotomy is performed first with the lungs deflated and then a right thoracotomy is performed. The pericardium is split open on both sides parallel and anterior to the phrenic nerve. The driveline is then passed through first, followed by implantation of the LVAD in the usual fashion. The outflow is subsequently passed across to the other side intrapericardially and anastomosed with the ascending aorta similar to above. The pericardium is repaired using a PTFE patch on both sides to prevent tension. The lungs are then re inflated and the chest closed over apical and basal drains.

\section{Advantages}

Transplants may take place at any time during the day or night whereas VAD implantation is usually performed during routine lists. Using the implantation technique where bilateral thoracotomy is carried out compared to our technique will avoid re-sternotomy during the night. A reciprocating saw may then be used on a virgin sternum at the time of transplantation rendering the re-entry process safer. Logically this is an ideal alternative method provided 


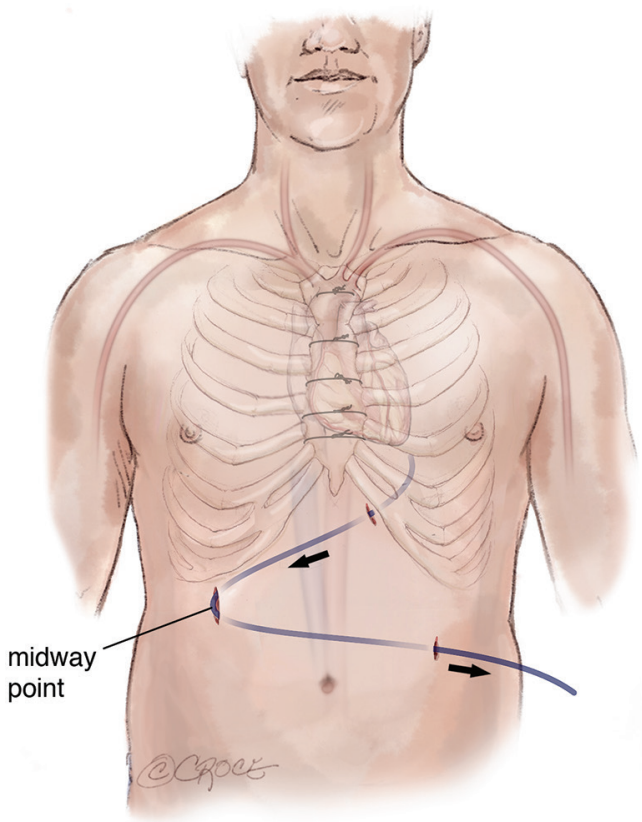

Figure 10 Path of the abdominal portion of the driveline. In cases where an extended tunnelled line is used by subcutaneous passage of the line, first to one side and then brought out of the skin on the opposite side, the driveline is explanted in two parts after making an incision on the skin at the half way point where it changes direction. The driveline is then pulled out with sustained force using a heavy clamp.

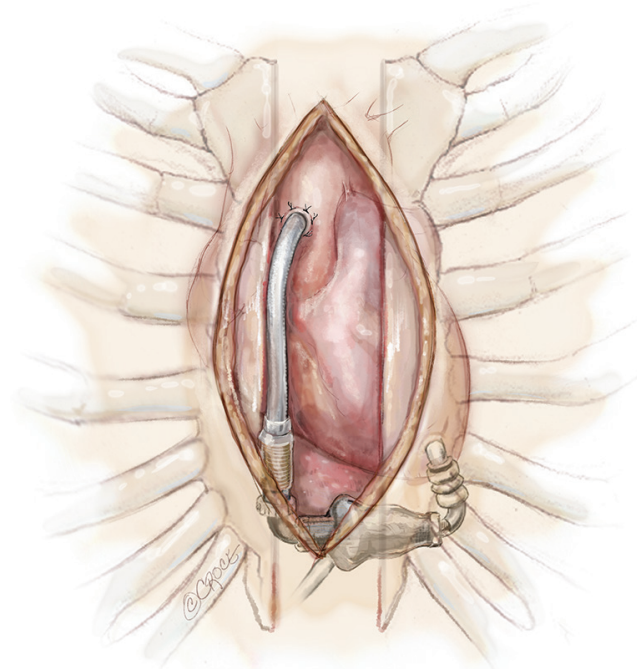

Figure $\mathbf{1 1}$ The driveline in the HeartMate II device is particularly vulnerable to injury at the time of re-sternotomy as it crosses the midline at the level of xiphoid process. the surgeon is familiar with the technique. From the point of view of the transplanting surgeon, apart from the sternotomy approach, explantation of the LVAD may follow a very similar sequence as described above.

\section{Caveats}

The possible disadvantages of the bilateral thoracotomy approach include management of post-thoracotomy pain compared with less painful sternotomy. In addition, this method does not eliminate formation of intra pericardial adhesions especially around the device however the adhesions may be less dense. This approach will also necessitate peripheral arterial cannulation for the totality of the procedure increasing the risk of leg ischaemia especially in smaller patients. The technique described in this review avoids a thoracotomy and necessitates less bypass time.

When the LVAD implantation has involved both a left thoracotomy and a limited proximal sternotomy instead of a right thoracotomy for anastomosis of the outflow tract to the ascending aorta (9), it is important to ascertain the position of the distal outflow tract in relation to the sternum as lateral placement of the anastomosis through this approach is more difficult.

It should be noted that the position of the driveline of the HeartMate II device renders it vulnerable during the explantation procedure. Of the different types of left ventricular assist devices, the HeartMate II left ventricular assist system by St Jude Medical was one of the first implantable devices of its kind to be widely used. The use of HVAD is now becoming more popular worldwide. HeartMate III is the most contemporary of these devices. Explantation of the latter two is almost identical. The major difference of HeartMate II as compared to HVAD or HeartMate III is in the position of the pump. The axial pump in HeartMate II is positioned in a subcutaneous pocket at the level of the left diaphragm extending to the midline at the lower end of the sternum. It is made of titanium so it would not be in danger of being injured during the explantation however the driveline exits the pump at a vulnerable position around the xiphoid process. Figure 11 illustrates the difference.

\section{Acknowledgements}

We would like to thank Anthony Garbutt for providing 
the photographs that formed the basis for the illustrations. Beth Croce for providing us with the illustrations and James Newton for providing and editing the video footage.

\section{Footnote}

Conflicts of Interest: The authors have no conflicts of interest to declare.

\section{References}

1. Holley CT, Harvey L, John R. Left ventricular assist devices as a bridge to cardiac transplantation. J Thorac Dis 2014;6:1110-9.

2. Amato JJ, Cotroneo JV, Galdieri RJ, et al. Experience with the polytetrafluoroethylene surgical membrane for pericardial closure in operations for congenital cardiac defects. J Thorac Cardiovasc Surg 1989;97:929-34.

3. Jacobs JP, Iyer RS, Weston JS, et al. Expanded PTFE membrane to prevent cardiac injury during resternotomy for congenital heart disease. Ann Thorac Surg 1996;62:1778-82.

Cite this article as: Khoshbin E, Schueler S. Pre-transplant ventricular assist device explant. Ann Cardiothorac Surg 2018;7(1):160-168. doi: 10.21037/acs.2018.01.04
4. Leprince P, Rahmati M, Bonnet N, et al. Expanded polytetrafluoroethylene membranes to wrap surfaces of circulatory support devices in patients undergoing bridge to heart transplantation. Eur J Cardiothorac Surg 2001;19:302-6.

5. Minale C, Nikol S, Hollweg G, et al. Clinical experience with expanded polytetrafluoroethylene Gore-Tex surgical membrane for pericardial closure: a study of 110 cases. J Card Surg 1988;3:193-201.

6. Vitali E, Russo C, Tiziano C, et al. Modified pericardial closure technique in patients with ventricular assist device. Ann Thorac Surg 2000;69:1278-9.

7. Copeland JG, Arabia FA, Smith RG, et al. Synthetic membrane neopericardium facilitates total artificial heart explantation. J Heart Lung Transplant 2001;20:654-6.

8. Popov AF, Hosseini MT, Zych B, et al. HeartWare left ventricular assist device implantation through bilateral anterior thoracotomy. Ann Thorac Surg 2012;93:674-6.

9. Hanke JS, Rojas SV, Avsar M, et al. Minimally-Invasive LVAD Implantation: State of the Art. Curr Cardiol Rev 2015;11:246-51. 